\title{
New astrometric measurement and reduction of USNO photographic observations of the main Saturnian satellites: 1974-1998*
}

\author{
V. Robert ${ }^{1,2}$, D. Pascu ${ }^{3, \star \star}$, V. Lainey ${ }^{2}$, J.-E. Arlot ${ }^{2}$, J.-P. De Cuyper ${ }^{4}$, V. Dehant ${ }^{4}$, and W. Thuillot ${ }^{2}$ \\ ${ }^{1}$ Institut Polytechnique des Sciences Avancées IPSA, 11-15 rue Maurice Grandcoing, 94200 Ivry-sur-Seine, France \\ e-mail: vincent.robert@obspm.fr \\ ${ }^{2}$ Institut de Mécanique Céleste et de Calcul des Éphémérides IMCCE, Observatoire de Paris, PSL Research University, CNRS, \\ Sorbonne Universités, UPMC Univ. Paris 06, Univ. Lille 1, 77 Av. Denfert-Rochereau, 75014 Paris, France \\ 3 United States Naval Observatory USNO, 3458 Massachusetts Ave NW, Washington, DC 20392, USA \\ 4 Royal Observatory of Belgium ROB, avenue Circulaire 3, 1180 Uccle, Belgique
}

Received 28 September 2016/ Accepted 12 October 2016

\begin{abstract}
Context. Accurate positional measurements of planets and satellites are used to improve our knowledge of their orbits and dynamics, and to infer the accuracy of the planet and satellite ephemerides.

Aims. In the framework of the European FP7 ESPaCE program, we provide the positions of Saturn and its main satellites taken with the US Naval Observatory 26-inch refractor from 1974 to 1998.

Methods. We measured 526 astrophotographic plates with the digitizer of the Royal Observatory of Belgium and reduced them through an optimal process that includes image, instrumental, and spherical corrections using the UCAC4 catalog to provide the most accurate equatorial (RA, Dec) positions.

Results. We compared the observed positions of the satellites with the theoretical positions from INPOP13c and DE432 planetary ephemerides and from NOE-6-2015-SAT and SAT375 satellite ephemerides. The mean post-fit rms residuals in equatorial positions range from \pm 68 mas for the Titan observations or $400 \mathrm{~km}$ at Saturn, to \pm 100 mas for the Hyperion observations or $600 \mathrm{~km}$ at Saturn. The mean post-fit rms intersatellite residuals range from \pm 46 mas for the Rhea-Titan observations or $280 \mathrm{~km}$ at $\mathrm{Saturn}$, to $\pm 72 \mathrm{mas}$ for the Hyperion-Titan observations or $430 \mathrm{~km}$ at Saturn.
\end{abstract}

Key words. astrometry - ephemerides - planets and satellites: individual: Saturn

\section{Introduction}

The European Satellite Partnership for Computing Ephemerides, the ESPaCE project, aims at strengthening the collaboration and at developing new knowledge, new technology, and products for the scientific community in the domains of the development of ephemerides and reference systems for natural satellites and spacecraft. Several European research centers involved in space sciences and dynamics were associated. The activity is focused on extracting and analyzing astrometric data from ground-based and spacecraft measurements that will be combined to build new ephemerides. It is intended to provide new dynamical models for natural satellites, a characterization of their rotation properties, and to improve spacecraft orbit determination from space science. Since we have demonstrated that a precise digitization and a new astrometric reduction of old photographic plates could provide very accurate positions (Robert 2011; Robert et al. 2011), the project leaders chose to

\footnotetext{
* Full Table 1 is only available at the CDS via anonymous ftp to cdsarc.u-strasbg.fr $(130.79 .128 .5)$ or via http://cdsarc.u-strasbg.fr/viz-bin/qcat?]/A+A/596/A37, at the Natural Satellites DataBase and Natural Satellites Data Center services of IMCCE via http://nsdb.imcce.fr/ or http://www . imcce. fr/nsdc/

$\star \star$ USNO retired.
}

consider such observations as a significant task for the ESPaCE program.

Recently, Desmars et al. (2009) have shown that astrometric data spread over a long time span were better than more accurate astrometric data spread over a short interval of time for dynamical and ephemeris purposes. The extrapolation of the ephemerides for space missions are more reliable. Similarly, Arlot et al. (2012) have shown the benefit of using old observations and how new reductions and future reductions with the Gaia catalog (Robin et al. 2012) will be useful. In view of this, we first obtained the large photographic plate archive of the Martian satellites taken at the US Naval Observatory (USNO) from 1967 to 1997 for remeasurement and reanalysis. Thanks to the accurate and dense star catalog used, and most important, thanks to the digitization of the photographic plates and the new astrometric treatment, we demonstrated that we were able to provide accurate positions for Mars and its satellites (Robert et al. 2014, 2015), and that astrometric data derived from photographic plates can compete with those of old spacecraft.

The success of this work and the new value of old photographic observations motivated us to study other planetary systems with such material. We have therefore obtained the large photographic plate archive of the Saturnian satellites taken at 
USNO from 1974 to 1998 and decided to reanalyze the complete series.

\section{Historical context}

In the early 1970s, as NASA was planning the "Grand Tour" of the outer planets, it was quickly determined that the existing ephemerides of the planetary satellites were not accurate enough for the reconnaissance of the Voyager probes with these bodies. NASA approached the USNO because of its institutional expertise in satellite studies for help in improving the orbits of the satellites. The Working Group of Outer Planet Satellites was formed under the leadership of USNO and NASA (Seidelmann 1977, 1979). Experts in the observation and dynamics of planetary satellites participated in workshops of the Working Group and included representatives from USNO, Greenwich Observatory in the UK, the Bureau des Longitudes (BDL) in Paris, the Jet Propulsion Laboratory (JPL), the University of Texas (McDonald Observatory), the University of Virginia (McCormick Observatory), the Harvard-Smithsonian Observatory, and the Lowell Observatory. The bulk of the photographic observations of the main satellites were made at USNO and the McCormick observatories, while the fainter satellites were observed at the McDonald observatory. Mutual event observations of the Galilean satellites were made principally by Lowell, Harvard-Smithsonian, and BDL staff. Theoretical studies and the adjustments of the satellite orbits to the observations were principally made at JPL and BDL. The final satellite orbits were of unprecedented accuracy and indispensable to the success of the Voyager Mission.

\section{USNO photographic observations}

Extensive and intensive visual micrometer observations of the Saturnian satellites were made from the late 19th century to the mid-20th century with the Alvan Clark great refractors. This included the observations by Hermann and Georg Struve with the Pulkovo 30-inch refractor and the observations of Barnard with the Yerkes 40-inch refractor. The most extensive series of visual observations of Saturn's satellites were made at USNO with the Clark 26-inch - the first of the great refractors, however. This series began shortly after the construction of the 26-inch in 1872 and ended in 1947 (Hammond 1911a,b; Hall \& Burton 1929, 1961).

The first long-focus photographic observations of the Saturnian satellites were made in 1926 by Alden \& O'Connell (1928) with the photographic refractor of the Yale Southern Station at Johannesburg, South Africa. Struve (1928) compared the Alden and O'Connell photographic observations to his own micrometer observations and concluded that the photographic observations were superior in accuracy, efficiency in observation, and reduction, and they served as a permanent record for future use. The Johannesburg series was continuous until 1935 and resumed in 1945 for three years. A shorter series was taken with the Thaw refractor of the Allegheny Observatory in Pittsburgh, Pennsylvania, from 1940 to 1947. This shorter series was taken to augment the South African series. Garcia (1972) discussed this combined set of photographic observations. However, these photographic observations had a major deficiency: the images of Mimas and Enceladus could not be measured. For this reason, USNO continued micrometer observations of the satellites until 1947.

The second and third of the extensive and intensive series of long-focus photographic observations were made concurrently at USNO and Pulkovo from the mid-1970s until the end of the century. The USNO photographic observations, taken with the 26-inch refractor, ended in 1999 when Kodak ceased making the necessary plates, while Pulkovo continued their photographic program for a few more years using ORWO plates (Kiseleva \& Khrutskaya 2007; Kiseleva et al. 2015).

Mimas, the innermost of Saturn's main satellites, had a poor observational history owing to its proximity to the rings and to its faintness. Enceladus, somewhat brighter and farther from the planet, was easier to observe. Since neither of these two could be measured on the Johannesburg or Allegheny plates, however, it was made a priority of the USNO observational program to obtain photographic positions for these two satellites. It was quickly determined that a 60 -s exposure with $103 \mathrm{aG}$ or 103aJ plates could record measurable images of Mimas at full aperture when the seeing and transparency were good, but only near elongation. Furthermore, under the finest seeing and transparency conditions, measurable images of Hyperion were obtained as well. It is not clear why the USNO visual refractor was successful in obtaining images of Mimas and Enceladus but the Allegheny's Thaw refractor and Yale's Johannesburg refractor were not. Both of these telescopes were photographic refractors with longer focal lengths. It may be that the emulsions of that period tended to bloom more easily than modern emulsions, or possibly the early plates were not anti-halation backed.

A second priority of the photographic program was to obtain photographic astrometric equatorial positions for Saturn. While meridian circle observations of the planets had improved in accuracy by obtaining their positions from the measurement of its satellites, the errors were still too high and only worked reasonably well for Jupiter and the Galilean moons. Meridian observations can only be made once in a night and during a shortened apparition (when observing the satellites). Photographic observations, however, can be made for a longer interval, and multiple observations can be made each night. It was therefore reasonable to expect that photographic observations would be a major improvement. To accomplish this, an accurate star catalog was necessary. It was expected that the AGK3 (Heckmann 1975) would serve that purpose.

Photographic observations of the satellites I-VIII of Saturn were begun at USNO in 1974, on the recommendation of the Working Group, to address the shortcomings of the satellite ephemerides. All plates in the series were taken with the USNO 26-inch refractor. Kodak 103aG, $5 \times 7 \times 0.06$ inch glass plates (anti-halation backed) were used in combination with a Schott GG14 (yellow) filter for observations made from 1975 to 1999. The 1974 series were taken on both $103 \mathrm{aG}$ and 103aJ plates. The GG14 filter was $5 \times 7$ inch $\times 3 \mathrm{~mm}$, polished optically flat and parallel. Placed immediately in front of the GG14 was a clear glass plate, $5 \times 7 \times 0.06$ inch, with a small partially transparent metallic Inconel filter, elliptical in shape, placed in its center by evaporation. The purpose of the small Inconel filter was to reduce the light of the planet to about tenth magnitude, preventing blooming of the planetary image and yielding a measurable image of the rings (see Figs. 1 and 2). A measurable image of the rings was sought to provide positions of the planet to compare with that determined from the satellites. A cartridge plate holder was fabricated in which the combination filter was fixed in the frame above the plate holder. In the observations, with the shutter closed and the plate holder in its cartridge fixed to the right of the focal plane, the Inconel filter was placed over the planet and the plate holder slid out of its cartridge and into the focal plane. The shutter was then opened and the exposure begun. The plate holder could be moved incrementally in the $x$-direction 
permitting several exposures on the plate. In this arrangement, the small Inconel filter was kept close to the emulsion (about $2 \mathrm{~mm}$ ) to reduce its penumbra and minimize the diffracted light around the edge of the filter. Two to three exposures were taken per plate. When Mimas was the target, 60-s exposures were taken at full aperture. When the equatorial position of Saturn was the intent, 30-s exposures were taken with the aperture diaphragmed to 16 inches. The purpose of the reduced aperture was to increase the f-ratio, increasing the "coma-free" field, and thus, increasing the available catalog stars used in the plate reduction to the plate corners. Finally, a trail of a nearby star was recorded on each plate, and occasional grating scale plates of the Pleiades and Praesepe clusters were taken for calibration (Pascu 1977, 1979).

The observations were continued until January 1999, when Kodak stopped the manufacture of photographic plates used in astrometry. Plates were taken at all oppositions except that of 1995 because of poor weather. In summary, 676 multiple exposure plates were taken on 177 nights over $25 \mathrm{yr}$ and 24 apparitions. The plates taken from 1974 to 1980 were measured with the USNO automatic measuring machine and reduced with the stars of the AGK3 catalog (Pascu \& Schmidt 1990). The rootmean square (rms) of the intersatellite observations ranged from \pm 70 mas for the Rhea-Titan observations to \pm 150 mas for the Mimas-Titan observations. The equatorial RA and Dec of Saturn were determined with a rms of \pm 250 mas, due mainly to the errors in the AGK3 catalog. These observations have been used in support of all reconnaissance probes to the Saturn system, as well as theoretical developments by dynamicists. Table A.1 summarizes the exposure data for the twenty-four oppositions in the series.

New developments in the past $20 \mathrm{yr}$ make the USNO plate archive valuable for reassessment. First, the plates from 1981 to 1998 have never been measured. Second, the new plate digitizer of the Royal Observatory of Belgium (de Cuyper et al. 2011) can make measurements of unprecedented accuracy. Third, the new star catalogs have improved tremendously in precision and density (Zacharias et al. 2013). The measurement and reduction of the entire twenty-four opposition USNO archive of plates of the Saturn system will be invaluable to the study of the motions and dynamics of that system (Lainey et al. 2015).

\section{Measurement and reduction}

Five hundred twenty-six plates were selected and transmitted to the ROB to be digitized (de Cuyper et al. 2011; Robert et al. 2011). Each plate contains two to three exposures shifted in the RA direction. The exposure times of the photographic plates are 20-90 s. The field of view is 57 arcmin on the $x$-axis and 43 arcmin on the $y$-axis. We were able to make measurements for 1269 positions of Saturn, 257 positions of Mimas, 342 positions of Enceladus, 531 positions of Tethys, 639 positions of Dione, 1126 positions of Rhea, 1185 positions of Titan, 101 positions of Hyperion, and 992 positions of Iapetus.

Figure 1 shows the center of the digitized (positive) USNO Saturnian plate No. 02013, which is a typical digitized image. This 60-s exposure of the Saturn system was taken with the USNO 26-inch refractor on 24 January 1975. A Kodak $103 \mathrm{aG}$ plate, in combination with a Schott GG14 filter, was used to limit the bandwidth of the exposure to the visual focal properties of the 26-inch lens. The small, partially transparent Inconel filter covers the planet, reducing its light and providing a measurable image of Saturn and the rings. The shadow of the Inconel filter is clearly seen around Saturn. The satellites visible in this exposure

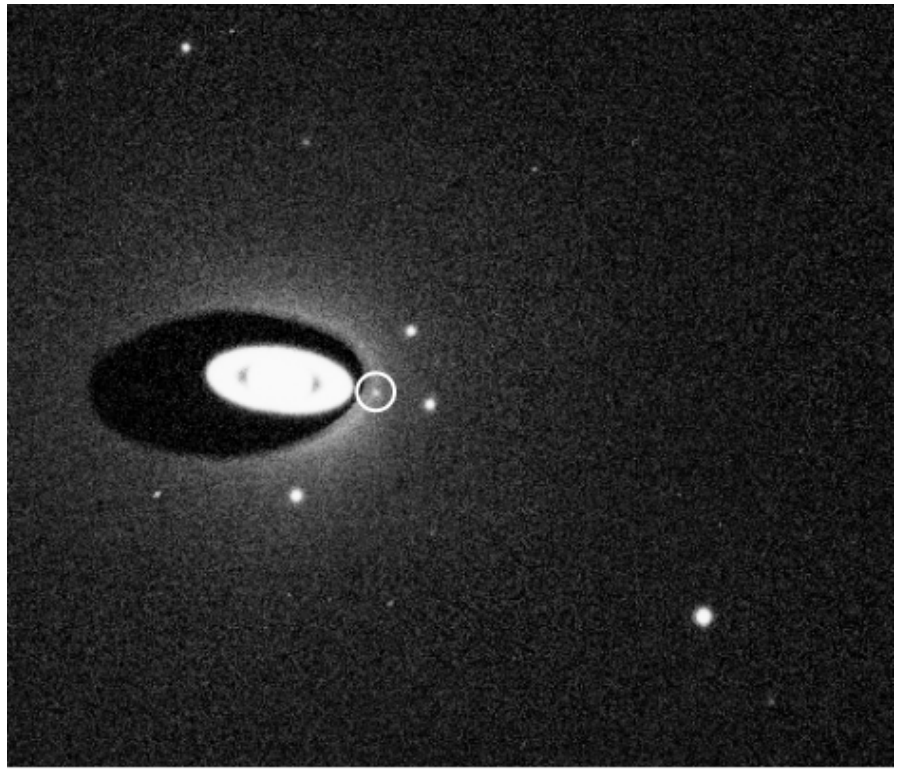

Fig. 1. Center of the digitization (positive) of the USNO 26-inch Saturnian plate No. 02013. Brighter images are the satellites. From left to right: Iapetus, Rhea, Mimas (displayed in the white circle), Dione, Tethys, and Titan. The dark shape around Saturn is the shadow of the small Inconel filter. Enceladus is close to Saturn, and behind the filter. North is up, east to the left.

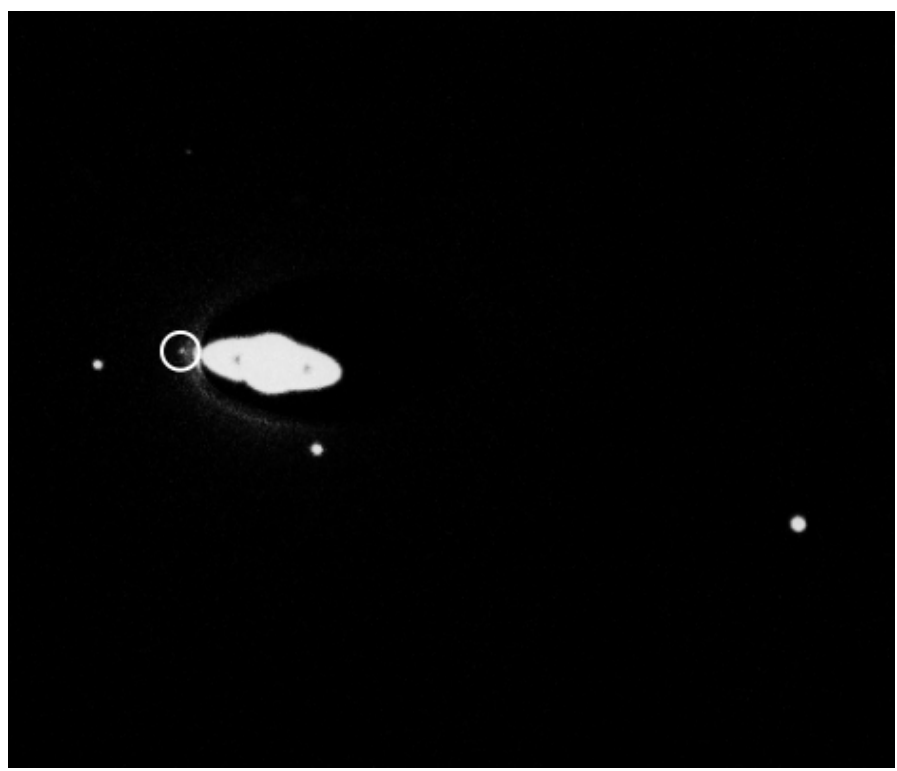

Fig. 2. Center of the digitization (positive) of the USNO 26-inch Saturnian plate No. 04017. From left to right: Dione, Mimas (displayed in the white circle), Rhea, and Titan. North is up, east to the left.

are, from left to right, Iapetus, Rhea, Mimas, Dione, Tethys, and Titan. Enceladus is behind the Inconel filter. North is up, east to the left.

Figure 2 shows the center of the digitized (positive) USNO Saturnian plate No. 04017, which is a typical digitized image. This 60-s exposure of the Saturn system was taken with the USNO 26-inch refractor on 16 March 1977. The plate/filter combination is the same as for Fig. 1. The satellites visible in this exposure are, from left to right, Dione, Mimas, Rhea, and Titan. North is up, east to the left.

Measurement and reduction techniques were the same as described in Robert et al. (2014) with small differences because 
the planet positions were determined by using the method described in Peng (2005). We fitted an ellipse to the edge of the outer rings by least squares to measure its geometric center. Measured $(x, y)$ plate positions were corrected for instrumental and spherical effects. The observations contain two to fifteen UCAC4 reference stars (Zacharias et al. 2013), and the reductions were performed using suitable four constant functional models to provide equatorial (RA, Dec) astrometric positions of the planet and its satellites. Scale $\rho$, orientation $\theta$, and offsets $\Delta_{x}$ and $\Delta_{y}$ were modeled for the determination of the tangential $(X, Y)$ coordinates. All our observations were equatorial (RA, Dec) astrometric positions obtained from tangential $(X, Y)$ coordinates by using the gnomonic inverse projection and determined in an ICRS geocentric reference frame to be easily compared with the most recent ephemerides.

\section{Positioning results}

We used our data to fit the numerical integration of the last IMCCE NOE-6-2015-SAT satellite ephemerides (Lainey et al. 2015). We rejected observations for which the astrometric calibration was not reliable, which mainly occurred when the shape of the reference stars due to errors in the guiding or their faintness did not allow for a precise measurement. We then focused on individual observations for which the residuals of the satellites were independently lower than the $3 \sigma$ value of their rms residuals in right ascension and declination, before final adjustment. Positions for Saturn were derived indirectly from the four bright moons.

We compared the positions of Saturn and its main satellites with their theoretical computed positions given by the INPOP13c planetary ephemeris (Fienga et al. 2014) and NOE6-2015-SAT satellite ephemerides. This concerns 1055 positions of Saturn, 123 positions of Mimas, 285 positions of Enceladus, 389 positions of Tethys, 524 positions of Dione, 849 positions of Rhea, 968 positions of Titan, 29 positions of Hyperion, and 779 positions of Iapetus. In the list available in electronic form at the CDS and IMCCE, the corresponding geocentric observed positions refer to the ICRF, and the mean time of observation is given in Barycentric Dynamical Time TDB. Starting from the lefthand column, we provide the object name, the mean TDB date of observation in Julian Days, the geocentric observed right ascension, and declination in degrees. Table 1 gives an extract of this list. The distributions of the $(\mathrm{O}-\mathrm{C}) \mathrm{s}$ and residuals in equatorial right ascension and declination are provided in Figs. 3-7, and Table 2. They show the difference of (RA, Dec) coordinates for individual planet and satellites, hence the observed positions versus positions calculated from INPOP13c ephemeris.

Offsets for each night of a single opposition set are small, but biases can occur in both the RA and Dec coordinates. We conclude that the exposure timing can be ruled out. The dome clock was calibrated each night to the USNO masterclock, and the camera shutter could be opened and closed on integral seconds with a precision of $0.2-0.3 \mathrm{~s}$. The time of mid-exposure thus has a precision better than 0.5 second. We have already discussed the impact of local systematic errors of the reference star catalogs (Robert 2011; Robert et al. 2011, 2014, 2015): the epoch differences between the plates and the central epoch of the reference stars are up to about $20 \mathrm{yr}$, and their expected systematic errors are about 10-50 mas. Observations of each opposition span one to six months, that is to say, from $1^{\circ}$ to $6^{\circ}$ along the path of Saturn. The sets of reference stars are different, and with about two to fifteen stars per field, the astrometric reduction may be affected by catalog errors of individual reference stars.
Table 1. Extract from the astrometric positions list of Saturn and its main satellites.

\begin{tabular}{cccc}
\hline \hline Object & Date $(\mathrm{TDB})$ & RA $(\mathrm{deg})$ & Dec $(\mathrm{deg})$ \\
\hline Saturn & 2442130.511344 & 88.568329 & 22.635496 \\
Mimas & 2442130.511344 & 88.561443 & 22.632991 \\
Enceladus & 2442130.511344 & 88.557874 & 22.633639 \\
Tethys & 2442130.511344 & 88.560271 & 22.639330 \\
Dione & 2442130.511344 & 88.551249 & 22.633259 \\
Rhea & 2450362.642617 & 4.027505 & -1.095570 \\
Titan & 2450362.642617 & 4.106022 & -1.101121 \\
Hyperion & 2450362.642617 & 4.008639 & -1.090307 \\
Iapetus & 2450362.642617 & 3.902936 & -1.114601 \\
\hline
\end{tabular}

Notes. The full table is available at the CDS and IMCCE.

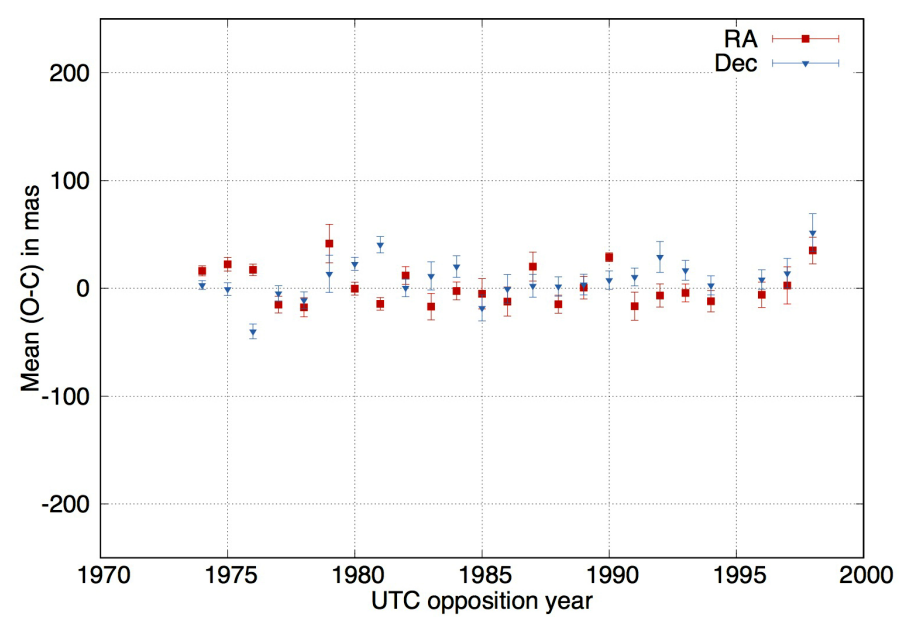

Fig. 3. Equatorial mean $(\mathrm{O}-\mathrm{C}) \mathrm{s}$ for Saturn positions according to INPOP13c ephemerides. The $x$-axis shows the UTC year of opposition and $y$-axis the mean $(\mathrm{O}-\mathrm{C})$ with errors. Red squares and blue triangles denote RA and Dec coordinates, respectively.

Offsets for each opposition set, visible in Figs. 4 and 6, are small and can be modeled with a $14.7 \pm 0.004$-yr periodic signal of $5.2 \pm 1.8$ mas of magnitude. This periodicity corresponds to both the favorable opposition period of Saturn and the edgewise presentation of the rings. Because this effect is similar to that we found with the analysis of the USNO Martian observations (Robert et al. 2015), however, this second case can be ruled out. We conclude that when the planet crosses the ecliptic plane, its relative distance to Earth is either minimum or maximum, and the offset is zero. This indicates that the eccentricity and inclination of the orbit of Saturn introduces a height effect as well. We also conclude that any mismodeling of instrumental and spherical corrections, and in particular, of the total and differential atmospheric refraction, can be ruled out because the offsets at minimum and maximum distances are zero, so that no effect remains. Moreover, the signal is eliminated while dealing with intersatellite residuals. We fitted a periodic signal with the residuals according to DE432 ephemeris (Folkner 2014) and the amplitude slightly changed to 4.2 mas, but observations used for the fit of the numerical integrations leading to INPOP13c and DE432 are quite similar. We also fitted a periodic signal according to older ephemerides for which observations used for the fit and numerical integrations are significantly different. The amplitude of the fitted signal according to DE200 ephemeris (Standish 1982) and VSOP87 ephemeris (Bretagnon \& Francou 1988) changed to 38.8 mas and 37.6 mas, 
Table 2. Details of the equatorial mean (O-C)s for Saturn and mean residuals for the satellites in mas, according to NOE-6-2015-SAT and INPOP13c ephemerides.

\begin{tabular}{ccccccc}
\hline \hline & $\overline{\Delta \alpha \cos \delta}$ & $\begin{array}{c}S E M_{\Delta \alpha \cos \delta} \\
+/-\end{array}$ & $\begin{array}{c}\sigma_{\Delta \alpha \cos \delta} \\
+/-\end{array}$ & $\overline{\Delta \delta}$ & $\begin{array}{c}S E M_{\Delta \delta} \\
+/-\end{array}$ & $\begin{array}{c}\sigma_{\Delta \delta} \\
+/-\end{array}$ \\
\hline Saturn & 0.5 & 2.0 & 63.5 & 2.2 & 2.0 & 64.3 \\
Mimas & 1.7 & 9.3 & 102.7 & -1.0 & 8.8 & 97.9 \\
Enceladus & 6.9 & 6.0 & 101.5 & -1.1 & 5.6 & 93.8 \\
Tethys & 1.8 & 4.0 & 79.1 & 4.1 & 4.0 & 78.2 \\
Dione & 2.1 & 3.3 & 75.5 & 4.1 & 3.3 & 75.6 \\
Rhea & 3.5 & 2.5 & 72.1 & 0.8 & 2.5 & 72.1 \\
Titan & 1.5 & 2.1 & 66.6 & 1.0 & 2.2 & 69.0 \\
Hyperion & -9.8 & 21.0 & 113.0 & -0.2 & 17.0 & 91.7 \\
Iapetus & 7.4 & 2.8 & 77.0 & -8.8 & 2.4 & 67.6 \\
\hline
\end{tabular}
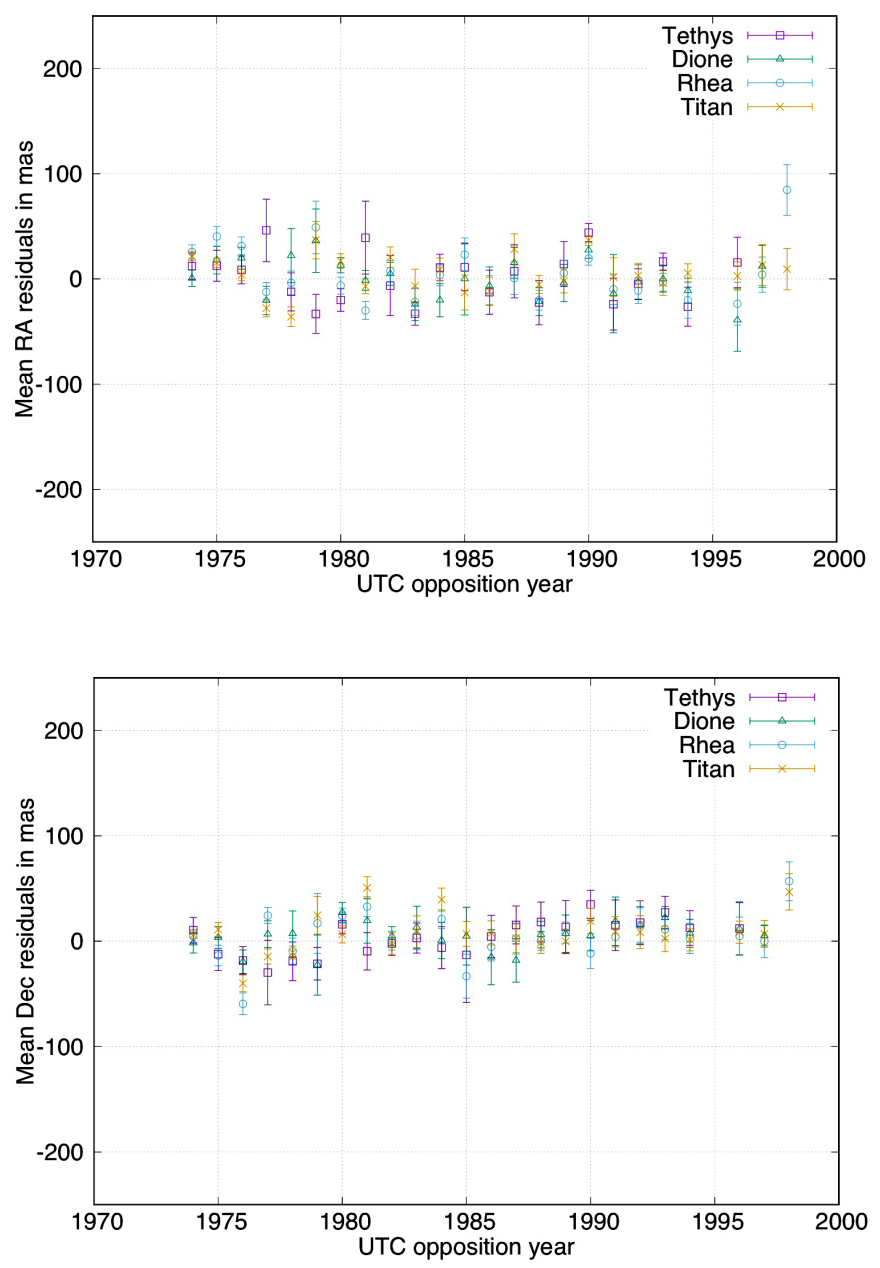

Fig. 4. Equatorial mean residuals according to NOE-6-2015-SAT and INPOP13c ephemerides. The $x$-axis shows the UTC year of opposition and $y$-axis the RA and Dec mean residuals with errors. Purple squares denote Tethys, green triangles Dione, blue circles Rhea, and yellow crosses Titan.

respectively. This confirms that adjustment and weighting of the planetary ephemeris used could explain the difference in the magnitude of the effect.

The average residual values for the observations made from 1974 to 1998 are very low in RA and Dec coordinates. We can see that the satellite variances in RA are higher than those in Dec, since their apparent motion is essentially in RA. To estimate the influence of the planetary ephemeris on the results, we computed

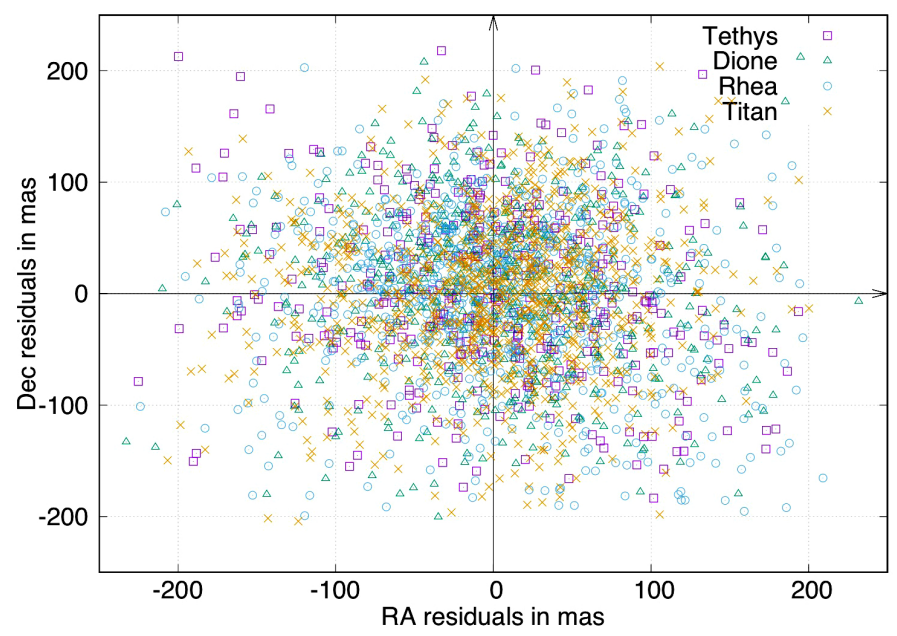

Fig. 5. Equatorial residuals according to NOE-6-2015-SAT and INPOP13c ephemerides. The $x$-axis shows the RA residuals and $y$-axis the Dec residuals. Purple squares denote Tethys, green triangles Dione, blue circles Rhea, and yellow crosses Titan.

the difference between observed positions and positions calculated from DE432 ephemeris and from older planetary models and, in particular, from DE421 (Folkner et al. 2009), DE200, INPOP08 (Fienga et al. 2009), and VSOP87 ephemerides. More precisely, data from the Cassini mission were introduced for the fit of DE421 and INPOP08, and it allowed us to correct the planet positions for important biases in the RA coordinate that are mainly due to imprecisions in its longitude, in its distances from Earth, and in its geocentric angular positions. Differences with DE200 and VSOP87 will let us appreciate such improvements. Table A.2 shows the difference between the observed positions and positions calculated from the corresponding ephemerides. The mean difference between INPOP13c, INPOP08, DE432, and DE421 for Saturn is supposed to be smaller than 3 mas (Fienga et al. 2014), and we may deduce from our results that this value is realistic for both coordinates. As we determined with the analysis of the USNO Martian observations, however, these average $(\mathrm{O}-\mathrm{C})$ values are minimized over our time span because a large number of observations were taken between successive oppositions of Saturn and the periodic evolution alternates between minimum and maximum values. Even though the differences between the most recent planetary ephemerides are small, we estimate from the amplitude of the periodic signals we fitted that the mean external error of DE432 is 4.2 mas, and 5.2 mas for INPOP13c. We have 

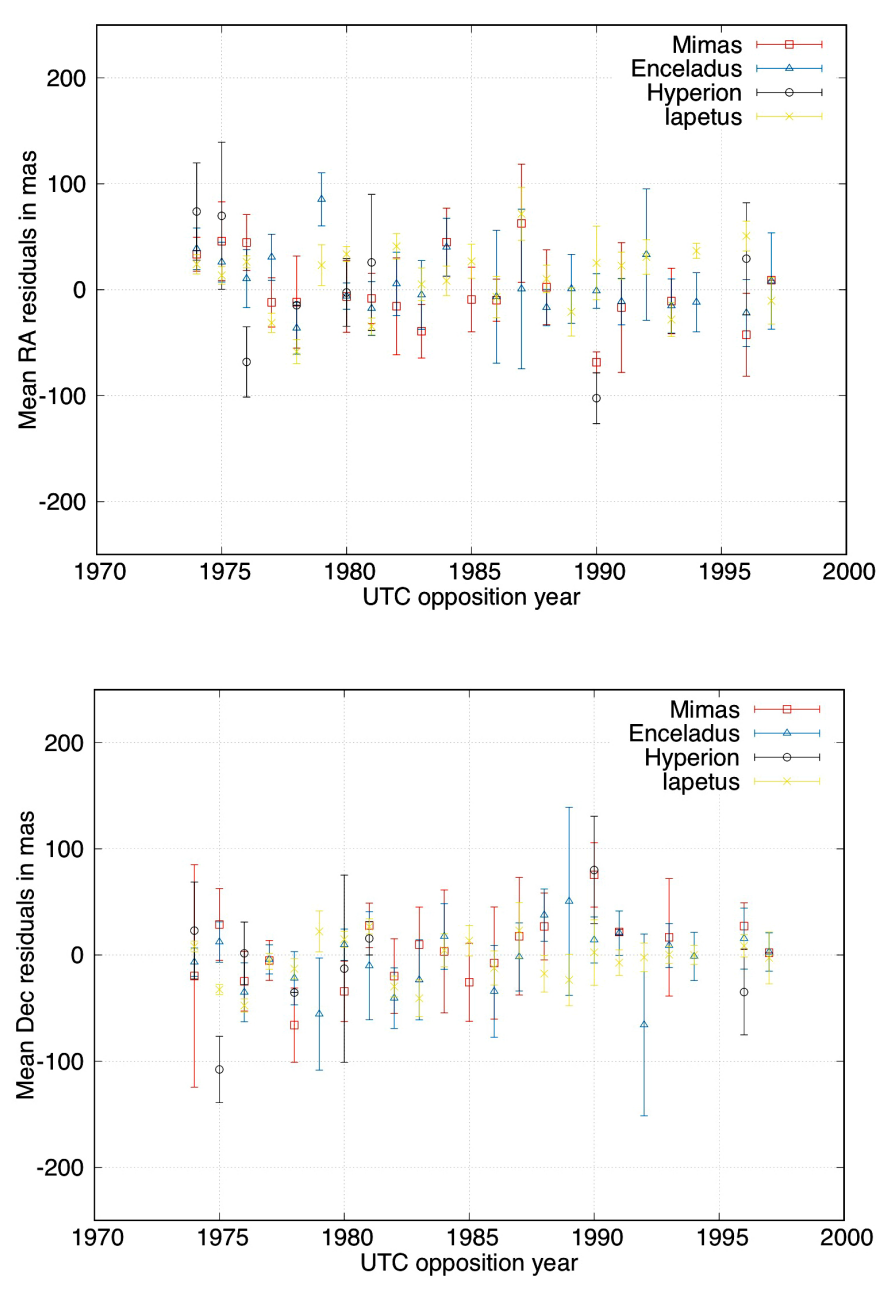

Fig. 6. Equatorial mean residuals according to NOE-6-2015-SAT and INPOP13c ephemerides. The $x$-axis shows the UTC year of opposition and $y$-axis the RA and Dec mean residuals with errors. Orange squares denote Mimas, blue triangles Enceladus, black circles Hyperion, and yellow crosses Iapetus.

already discussed the impact of accidental observational errors and mismodeling, and we assume that no observational effect remains at the level of 1 mas (Kaplan et al. 1989; Robert 2011). As we concluded before, such a signal may be introduced by the use of the planetary model itself. Because only differences between planetary models are evaluated in corresponding papers, we have no information about their external errors that are generally estimated separately by comparison between observations that were not used for the fit and the theories. We there see from old observations that the mean external error of recent planetary ephemerides for Saturn could reach 5 mas, that is to say, twice the internal accuracy officially provided. We previously found such a bias with the Martian system (Robert et al. 2015). Comparisons with old observations indicate that the external error of recent planetary ephemerides for Mars reaches 10 mas. Further investigations are planned as well to confirm a preliminary result that indicates that the external error of most recent planetary models for Jupiter is up to 20 mas.

We also note that the amplitude of the fitted signal according to DE200 and VSOP87 ephemerides reached 38.7 mas and 37.6 mas, respectively. The main difference between DE200 and VSOP87 theories and planetary ephemerides built afterward is the introduction of data from the Cassini mission for the fit of Saturn. This permitted us to significantly improve our knowledge

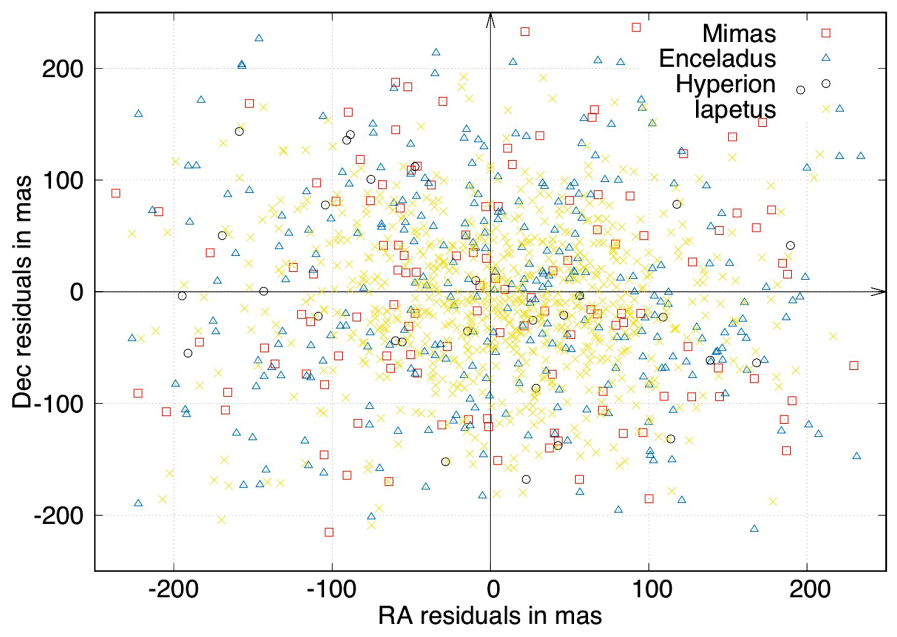

Fig. 7. Equatorial residuals according to NOE-6-2015-SAT and INPOP13c ephemerides. The $x$-axis shows the RA residuals and $y$-axis the Dec residuals. Orange squares denote Mimas, blue triangles Enceladus, black circles Hyperion, and yellow crosses Iapetus.

of its orbit. Our results, and especially the biases in the RA coordinates visible for these two models in Table A.2, are in agreement with the difference of 100 mas between fits with and without the Cassini data estimated by Fienga et al. (2009).

We finally compared the observed positions of the main Saturnian satellites with their theoretical positions given by the most recent SAT375 JPL ephemerides (Jacobson 2015). Results are quite similar to those given by NOE-6-2015-SAT model within 3 mas difference for all satellites except for Hyperion (15 mas difference in declination), which is consistent with the expected values. Table 3 shows the difference between the observed positions and positions calculated from SAT375 ephemerides. It also shows the difference with NOE-6-2011-SAT ephemerides (Lainey et al. 2012) for which Cassini data were not introduced.

The key point is that the NOE-6-2015-SAT/INPOP13c rms $(\mathrm{O}-\mathrm{C})$ for all observations of Saturn is 63.9 mas. The NOE6-2015-SAT/INPOP13c rms residual for all observations is 100.3 mas for Mimas, 97.6 mas for Enceladus, 78.6 mas for Tethys, 75.5 mas for Dione, 72.1 mas for Rhea, 67.8 mas for Titan, 102.3 mas for Hyperion, and 72.3 mas for Iapetus. These average rms $(\mathrm{O}-\mathrm{C}) \mathrm{s}$ and residuals correspond to our observation accuracies over twenty-four oppositions in the series. Pascu \& Schmidt (1990) previously measured and reduced exposures of Saturn and satellites taken from 1974 to 1980, mainly to estimate the precision of new photographic techniques. Photographic observations were compared to DE125 ephemeris (Standish 1985), and the external error in right ascension was \pm 200 mas, and in declination \pm 270 mas. These results were in agreement with what was expected, and the error due to the reference stars in the budget could explain the orders of magnitude. Moreover, a systematic error in declination was noted. Because no error due to the photographic observations could be found, it was suggested that it was introduced by the use of DE125 model itself, and in more detail, because of the biased visual transit circle observations that were used for its fit. The main counterparts of the errors were corrected with DE200 ephemerides and the introduction of other observations for the fit of Saturn, but biases in right ascensions remained this time, as we can see in Table A.2. These remaining errors were corrected with DE421 and INPOP08 ephemerides afterward. 
Table 3. Details of the equatorial (O-C)s in mas according to SAT375, NOE-6-2011-SAT, and INPOP13c ephemerides.

\begin{tabular}{ccccccc}
\hline \hline & $\overline{(\mathrm{O}-\mathrm{C})_{\alpha \cos \delta}}$ & $\begin{array}{c}S E M_{\alpha \cos \delta} \\
+/-\end{array}$ & $\begin{array}{c}\sigma_{\alpha \cos \delta} \\
+/-\end{array}$ & $\begin{array}{c}\overline{(\mathrm{O}-\mathrm{C})_{\delta}} \\
\begin{array}{c}S E M_{\delta} \\
+/-\end{array}\end{array} \begin{array}{c}\sigma_{\delta} \\
+/-\end{array}$ \\
\hline SAT375 & 1.7 & 9.3 & 103.1 & -0.1 & 8.8 & 98.1 \\
Mimas & 6.4 & 6.0 & 101.5 & -1.3 & 5.6 & 93.8 \\
Enceladus & 1.0 & 4.0 & 78.7 & 4.4 & 3.9 & 77.7 \\
Tethys & 2.0 & 3.3 & 75.4 & 4.0 & 3.3 & 75.5 \\
Dione & 3.3 & 2.5 & 72.0 & 0.7 & 2.5 & 72.0 \\
Rhea & 3.4 & 2.1 & 66.5 & 1.4 & 2.2 & 68.9 \\
Titan & -13.8 & 20.6 & 113.0 & 14.8 & 17.7 & 96.8 \\
Hyperion & 6.9 & 2.7 & 76.2 & -8.7 & 2.4 & 67.6 \\
Iapetus & & & & & & \\
NOE-6-2011-SAT & 1.3 & 9.2 & 102.2 & -2.5 & 8.8 & 98.0 \\
Mimas & 7.5 & 6.0 & 101.3 & -1.3 & 5.6 & 93.7 \\
Enceladus & 2.7 & 4.1 & 80.1 & 5.0 & 4.0 & 78.3 \\
Tethys & 2.7 & 3.3 & 75.7 & 5.2 & 3.3 & 76.0 \\
Dione & 3.8 & 2.5 & 72.8 & 0.5 & 2.5 & 71.9 \\
Rhea & 2.4 & 2.2 & 67.3 & 1.7 & 2.2 & 68.6 \\
Titan & -7.7 & 21.2 & 114.0 & 0.3 & 17.1 & 92.0 \\
Hyperion & -5.3 & 2.7 & 75.7 & -11.5 & 2.5 & 69.8 \\
Iapetus & & & & & &
\end{tabular}

\section{Intersatellite observations}

With Fig. 8 we investigate the effect of the signal-to-noise ratio $(\mathrm{S} / \mathrm{N})$ on the precision of the observations. The mean (satellite - Titan) rms residuals in $(\Delta \alpha \cos \delta, \Delta \delta)$ coordinates are plotted against the (satellite - Titan) $\Delta V$ magnitude difference as a measure of $\mathrm{S} / \mathrm{N}$. The solid line fitted to our data demonstrates the decline in positional precision with the faintness of the satellite images. While in general, the fainter satellites have less well determined orbits than the bright ones, the residuals have Gaussian distributions and are not systematic. They therefore represent observational error, including unmodeled representational errors (such as seeing), measuring errors, plate modeling errors, and catalog reference star errors. We compared our results to the dashed line that is taken from an earlier analysis by Pascu (1994). In that analysis, the plate material from 1974 to 1980 was measured with the USNO automatic measuring machine and reduced with stars from the AGK3 catalog. The rms residuals of these observations resulted from orbital adjustments at the British Almanac Office. The question arises as to what caused the difference in the slope of the relation. Assuming the quality of the early plates to be the same as that of the entire archive, we can draw some conclusions. Since the plates are the same, the difference in slope is not due to representational errors. Moreover, since the lines cross at the magnitude of Titan, catalog errors and plate modeling errors are probably not the cause. Our interpretation is that the scanning of the images and their centroiding is much superior in the measurement of faint images than with the older automatic measuring machines.

With Fig. 9 we investigate the astrometric limitation in external precision due to the atmosphere. Lindegren (1980) discussed the wave-front distortion due to the atmospheric turbulence and its influence on astrometry. He concluded that the mean error of the measured angle $\theta$ (radians) between two objects near the zenith is given in arcsec by

$m . e=1.3 \theta^{0.25} T^{-0.5}$

where $T \gg 300 \theta$ is the exposure time in seconds. We plotted rms residuals in the (satellite - Titan) separations against the separations for Tethys, Dione and Rhea for the 50-60 s exposures. For

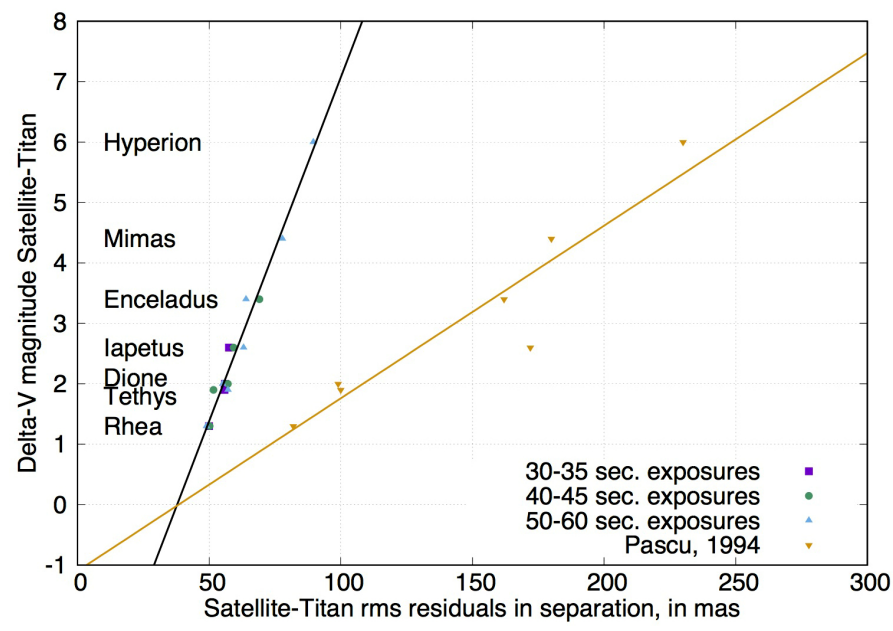

Fig. 8. rms residuals in separation from Titan according to NOE-62015-SAT ephemerides, with regard to the exposure time. The $x$-axis shows the mean rms residuals in separation and $y$-axis the difference in $V$-magnitude from Titan. Purple squares denote $30-35 \mathrm{~s}$ exposures, green circles 40-45 s exposures, and blue triangles 50-60 s exposures. Orange triangles denote previous measurements made by Pascu (1994).

comparison, we also plotted Lindegren's function above. The curves for all three satellites are nearly coincident, but about 15 mas above the Lindegren function. There appears to be an excess variance in the separations. An obvious source of such an error is the motion of the satellites relative to the sidereal rate during these unguided exposures. This may result in a systematic magnitude error in the (satellite - Titan) separations since Titan is about 2 mag brighter than the other three. However, this would manifest itself as an accidental error because of the multiple configurations of each satellite relative to Titan. On the other hand, the Lindegren function, which is referenced to the zenith, would not apply exactly to these observations, which were made on the ecliptic and at times at large zenith distances. This is more likely the source of the small excess error in the observations, and we conclude that we have reached the limit in precision for these 


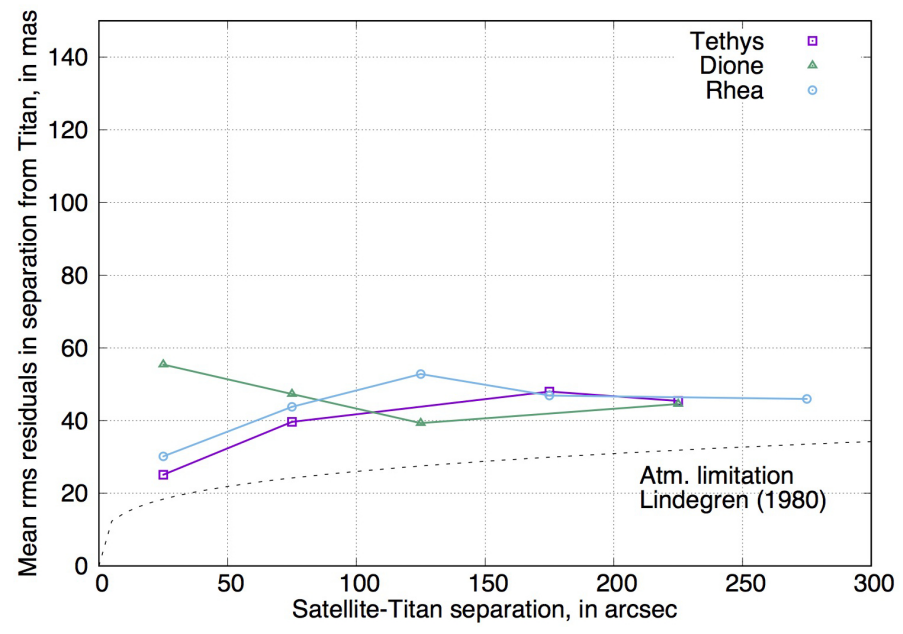

Fig. 9. Mean rms residuals in separation from Titan according to NOE6-2015-SAT ephemerides, for the 50-60 s exposures. The $x$-axis shows the satellite-Titan separation and the $y$-axis the mean rms residuals in separation. Purple squares denote Tethys, green triangles Dione, and blue circles Rhea. Atmospheric limitation is given by Lindegren (1980).

observations based on the UCAC4, but we expect a significant improvement when we reduce these observations anew with the Gaia catalog.

\section{Summary and future work}

We analyzed a full series of astrophotographic plates of the main Saturnian satellites (I-VIII) taken at USNO from 1974 to 1998. We distinguished two main factors that contributed to the increase in precision: the digitization, and the new astrometric reduction. The digitization of the photographic plates now makes it possible to measure images with a high accuracy and to identify all the available stars, including those that are not detected by eye. In addition, a new astrometric reduction with an accurate and dense star catalog makes it possible to correct for all instrumental and spherical effects, and to decrease the number of unknown parameters. Thus, we were able to provide astrometric (RA, Dec) positions of the satellites, allowing us to deduce positions of Saturn indirectly, with overall $\mathrm{rms}$ residuals of about 73 mas for the four brightest main satellites, and 93 mas for the four faintest main satellites.

These results confirm the high value in continuing the reduction of old observations, and photographic plates in particular. We also look forward to the arrival of the complete Gaia reference star catalog and its proper motions especially, because new reductions will yield increased accuracy by eliminating any errors caused by reference star positions. We expect significant improvement in the equatorial positions.

Because most of the observatories and national archives have old and useful observations, we have started to establish contacts for scientific purposes, and more particularly, to improve the dynamics of the planetary systems with old data, in the framework of the FP7 ESPaCE program. We now continue in the framework of the NAROO project ${ }^{1}$. A new sub-micrometric digitizer will be set up in Paris at the end of 2016, with a new center of digitization and analysis of old observations. It is intended to digitize old astrophotographic plates with high accuracy, reanalyze the observations, produce new (RA, Dec) astrometric positions of the objects, and provide a free database for the community. Our main goal is to provide accurate positions of the planets, satellites, asteroids and comets over one century.

Acknowledgements. The research leading to these results has received funding from the European Community's Seventh Framework Program (FP7/2007-2013) under grant agreement No. 263466 for the FP7-ESPaCE program, the International Space Science Institute ISSI, and the CNRS/INSU/CNES "Programme National de Planétologie" PNP and "Gravitation, Références, Astronomie, Métrologie" GRAM programs. The authors are indebted to all participants of the Encelade WG.

\section{References}

Alden, R. L., \& O’Connell, W. 1928, AJ, 38, 53

Arlot, J.-E., Desmars, J., Lainey, V., \& Robert, V. 2012, Planet. Space Sci., 73, 66

Bretagnon, P., \& Francou, G. 1988, A\&A, 202, 309

de Cuyper, J.-P., de Decker, G., Winter, L., \& Zacharias, N. 2011, in ASP Data Analysis Software and Systems XX, eds. I. N. Evans, A. Accomazzi, D. J. Mink, \& A. H. Rots, ASP Conf. Ser., 442, 301

Desmars, J., Arlot, S., Arlot, J.-E., Lainey, V., \& Vienne, A. 2009, A\&A, 499, 321

Fienga, A., Laskar, J., Morley, T., et al. 2009, A\&A, 507, 1675

Fienga, A., Manche, H., Laskar, J., Gastineau, M., \& Verna, A. 2014, IMCCE, Observatoire de Paris [arXiv: 1405. 0484]

Folkner, W. M. 2014, Jet Propulsion Laboratory Memorandum 392R-14-003

Folkner, W. M., Williams, J. G., \& Boggs, D. H. 2009, Interplanetary Network Progress Report, 178, 1

Garcia, H. A. 1972, AJ, 77, 684

Hall, Jr., A., \& Burton, H. E. 1929, Publications of the US Naval Observatory, Second Series, XII, 92

Hall, Jr., A., \& Burton, H. E. 1961, Publications of the US Naval Observatory, Second Series, Part III, XVII

Hammond, J. C. 1911a, Publications of the US Naval Observatory, Second Series, VI, A15

Hammond, J. C. 1911b, AJ, 26, 175

Heckmann, O. 1975, AGK 3, Star catalogue of positions and proper motions north of -2.5 deg declination (Hamburg-Bergedorf: Hamburger Sternwarte) Jacobson, R. A. 2015, NASA NAIF Generic Kernels

Kaplan, G. H., Hughes, J. A., Seidelmann, P. K., Smith, C. A., \& Yallop, B. D. 1989, AJ, 97, 1197

Kiseleva, T. P., \& Khrutskaya, E. V. 2007, Sol. Syst. Res., 41, 72

Kiseleva, T. P., Vasil'eva, T. A., Izmailov, I. S., \& Roshchina, E. A. 2015, Sol. Syst. Res., 49, 72

Lainey, V., Karatekin, Ö., Desmars, J., et al. 2012, ApJ, 752, 14

Lainey, V., Jacobson, R. A., Tajeddine, R., et al. 2015, ArXiv e-prints [arXiv: 1510.05870$]$

Lindegren, L. 1980, A\&A, 89, 41

Pascu, D. 1977, in Planetary Satellites, ed. J. A. Burns, IAU Colloq. 28, 63

Pascu, D. 1979, in Natural and Artificial Satellite Motion, eds. P. E. Nacozy, \& S. Ferraz-Mello, 17

Pascu, D. 1994, in Galactic and Solar System Optical Astrometry, eds. L. V. Morrison, \& G. F. Gilmore, 304

Pascu, D., \& Schmidt, R. E. 1990, AJ, 99, 1974

Peng, Q. Y. 2005, MNRAS, 359, 1597

Robert, V. 2011, Ph.D. Thesis, Observatoire de Paris

Robert, V., de Cuyper, J.-P., Arlot, J.-E., et al. 2011, MNRAS, 415, 701

Robert, V., Lainey, V., Pascu, D., et al. 2014, A\&A, 572, A104

Robert, V., Lainey, V., Pascu, D., et al. 2015, A\&A, 582, A36

Robin, A. C., Luri, X., Reylé, C., et al. 2012, A\&A, 543, A100

Seidelmann, P. K. 1977, in Planetary Satellites, ed. J. A. Burns, IAU Colloq. 28, 533

Seidelmann, P. K. 1979, in Natural and Artificial Satellite Motion, eds. P. E. Nacozy, \& S. Ferraz-Mello, 3

Standish, Jr., E. M. 1982, A\&A, 114, 297

Standish, Jr., E. M. 1985, Jet Propulsion Laboratory Memorandum, 314.6

Struve, G. 1928, AJ, 39, 9

Zacharias, N., Finch, C. T., Girard, T. M., et al. 2013, AJ, 145, 44

\footnotetext{
1 See the NAROO webpage of IMCCE and Paris Observatory at http://nsdb.imcce.fr/naroo/
} 
V. Robert et al.: USNO astrometric observations of Saturn

\section{Appendix A: Additional tables}

Table A.1. Raw statistics of the USNO Saturnian photographic plates that were digitized at the ROB.

\begin{tabular}{|c|c|c|c|c|c|}
\hline Series & Opposition & Month & Emulsion & Exp. time (s) & Observations \\
\hline 01 & 1974 & $2-3$ & $103 \mathrm{aG}-103 \mathrm{aJ}$ & $30-90$ & 80 \\
\hline 02 & 1975 & $1,3-4$ & $103 \mathrm{aG}$ & $30-90$ & 78 \\
\hline 03 & 1976 & $2-3$ & $103 \mathrm{aG}$ & $30-90$ & 114 \\
\hline 04 & 1977 & $2-4$ & $103 \mathrm{aG}$ & $30-60$ & 148 \\
\hline 05 & 1978 & $3-4$ & $103 \mathrm{aG}$ & $30-90$ & 63 \\
\hline 06 & 1979 & $3-4$ & $103 \mathrm{aG}$ & $21-60$ & 20 \\
\hline 07 & 1980 & 12 (1979), 1-6 & $103 \mathrm{aG}$ & $20-80$ & 129 \\
\hline 08 & 1981 & $1-3,5-6$ & $103 \mathrm{aG}$ & $30-60$ & 66 \\
\hline 09 & 1982 & $4-6$ & $103 \mathrm{aG}$ & $30-60$ & 57 \\
\hline 10 & 1983 & $3-6$ & $103 \mathrm{aG}$ & $30-63$ & 36 \\
\hline 11 & 1984 & $4-6$ & $103 \mathrm{aG}$ & $30-60$ & 54 \\
\hline 12 & 1985 & $5-8$ & $103 \mathrm{aG}$ & $30-60$ & 48 \\
\hline 13 & 1986 & $5-6$ & $103 \mathrm{aG}$ & $30-60$ & 93 \\
\hline 14 & 1987 & $6-8$ & $103 \mathrm{aG}$ & $30-90$ & 57 \\
\hline 15 & 1988 & $6-8$ & $103 \mathrm{aG}$ & $30-60$ & 99 \\
\hline 16 & 1989 & $6-9$ & $103 \mathrm{aG}$ & $30-90$ & 47 \\
\hline 17 & 1990 & $7-8,10$ & $103 \mathrm{aG}$ & $25-60$ & 30 \\
\hline 18 & 1991 & $7-10$ & $103 \mathrm{aG}$ & $30-90$ & 36 \\
\hline 19 & 1992 & $7,9-10$ & $103 \mathrm{aG}$ & $35-80$ & 32 \\
\hline 20 & 1993 & $7-10$ & $103 \mathrm{aG}$ & $40-60$ & 54 \\
\hline 21 & 1994 & $10-11$ & $103 \mathrm{aG}$ & $30-60$ & 24 \\
\hline 22 & 1996 & 10 & $103 \mathrm{aG}$ & $30-60$ & 27 \\
\hline 23 & 1997 & $11-12$ & $103 \mathrm{aG}$ & $30-60$ & 27 \\
\hline \multirow[t]{2}{*}{24} & 1998 & 10 & $103 \mathrm{aG}$ & 30 & 3 \\
\hline & & & & Total & 1422 \\
\hline
\end{tabular}


Table A.2. Details of the equatorial mean (O-C)s for Saturn and mean residuals for the satellites, in mas, according to NOE-6-2015-SAT and DE432, DE421, DE200, INPOP08, and VSOP87 ephemerides.

\begin{tabular}{|c|c|c|c|c|c|c|}
\hline & $\overline{\Delta \alpha \cos \delta}$ & $\begin{array}{c}\operatorname{SEM}_{\Delta \alpha \cos \delta} \\
+/-\end{array}$ & $\begin{array}{c}\sigma_{\Delta \alpha \cos \delta} \\
+/-\end{array}$ & $\overline{\Delta \delta}$ & $\begin{array}{c}\operatorname{SEM}_{\Delta \delta} \\
+/- \\
\end{array}$ & $\begin{array}{l}\sigma_{\Delta \delta} \\
+/-\end{array}$ \\
\hline \multicolumn{7}{|l|}{ DE432 } \\
\hline Saturn & 1.0 & 2.0 & 63.5 & 2.2 & 2.0 & 64.3 \\
\hline Mimas & 2.3 & 9.3 & 102.8 & -0.9 & 8.8 & 97.9 \\
\hline Enceladus & 7.5 & 6.0 & 101.6 & -1.1 & 5.6 & 93.8 \\
\hline Tethys & 2.4 & 4.0 & 79.1 & 4.1 & 4.0 & 78.2 \\
\hline Dione & 2.7 & 3.3 & 75.5 & 4.1 & 3.3 & 75.6 \\
\hline Rhea & 4.0 & 2.5 & 72.1 & 0.8 & 2.5 & 72.1 \\
\hline Titan & 2.0 & 2.1 & 66.6 & 1.1 & 2.2 & 69.0 \\
\hline Hyperion & -9.2 & 21.0 & 113.1 & -0.4 & 17.0 & 91.8 \\
\hline Iapetus & 8.0 & 2.8 & 77.0 & -8.8 & 2.4 & 67.7 \\
\hline \multicolumn{7}{|l|}{ DE421 } \\
\hline Saturn & 1.0 & 2.0 & 63.5 & 2.1 & 2.0 & 64.2 \\
\hline Mimas & 2.2 & 9.3 & 102.8 & -1.0 & 8.8 & 97.9 \\
\hline Enceladus & 7.5 & 6.0 & 101.5 & -1.1 & 5.6 & 93.7 \\
\hline Tethys & 2.3 & 4.0 & 79.1 & 4.0 & 4.0 & 78.1 \\
\hline Dione & 2.6 & 3.3 & 75.5 & 4.1 & 3.3 & 75.6 \\
\hline Rhea & 4.0 & 2.5 & 72.1 & 0.7 & 2.5 & 72.1 \\
\hline Titan & 2.0 & 2.1 & 66.6 & 1.0 & 2.2 & 68.9 \\
\hline Hyperion & -9.2 & 21.0 & 113.0 & 0.1 & 17.0 & 91.6 \\
\hline Iapetus & 7.9 & 2.8 & 77.0 & -8.8 & 2.4 & 67.6 \\
\hline \multicolumn{7}{|l|}{ DE200 } \\
\hline Saturn & -131.3 & 3.0 & 97.2 & 23.9 & 2.3 & 76.3 \\
\hline Mimas & -100.1 & 9.4 & 104.4 & 19.2 & 8.7 & 97.0 \\
\hline Enceladus & -80.7 & 6.4 & 108.9 & 13.2 & 5.7 & 96.2 \\
\hline Tethys & -102.0 & 4.8 & 94.7 & 18.5 & 4.2 & 82.5 \\
\hline Dione & -102.9 & 4.1 & 93.0 & 23.8 & 3.6 & 83.2 \\
\hline Rhea & -107.4 & 3.2 & 94.1 & 18.6 & 2.8 & 82.8 \\
\hline Titan & -109.9 & 2.6 & 82.3 & 21.3 & 2.6 & 80.5 \\
\hline Hyperion & -78.6 & 23.2 & 120.8 & 7.9 & 19.7 & 102.3 \\
\hline Iapetus & -97.0 & 3.2 & 89.9 & 13.7 & 2.7 & 74.7 \\
\hline \multicolumn{7}{|l|}{ INPOP08 } \\
\hline Saturn & 0.9 & 2.0 & 63.6 & 0.9 & 2.0 & 64.1 \\
\hline Mimas & 2.0 & 9.3 & 102.8 & -2.5 & 8.8 & 98.1 \\
\hline Enceladus & 7.3 & 6.0 & 101.6 & -2.0 & 5.6 & 93.8 \\
\hline Tethys & 2.2 & 4.0 & 79.2 & 2.8 & 4.0 & 78.1 \\
\hline Dione & 2.6 & 3.3 & 75.6 & 2.9 & 3.3 & 75.5 \\
\hline Rhea & 3.9 & 2.5 & 72.2 & -0.5 & 2.5 & 72.0 \\
\hline Titan & 1.9 & 2.1 & 66.6 & -0.3 & 2.2 & 68.8 \\
\hline Hyperion & -9.3 & 21.0 & 112.6 & -1.5 & 17.0 & 91.3 \\
\hline Iapetus & 7.8 & 2.8 & 77.0 & -9.8 & 2.4 & 67.7 \\
\hline \multicolumn{7}{|l|}{ VSOP87 } \\
\hline Saturn & -130.9 & 2.7 & 87.1 & 21.9 & 2.2 & 72.7 \\
\hline Mimas & -98.4 & 8.7 & 96.7 & 18.7 & 8.7 & 96.6 \\
\hline Enceladus & -88.5 & 6.1 & 103.8 & 11.9 & 5.6 & 94.6 \\
\hline Tethys & -111.0 & 4.6 & 90.5 & 16.2 & 4.1 & 80.5 \\
\hline Dione & -127.6 & 3.8 & 86.9 & 21.3 & 3.5 & 80.5 \\
\hline Rhea & -110.8 & 3.0 & 86.9 & 17.4 & 2.7 & 79.9 \\
\hline Titan & -112.2 & 2.4 & 75.8 & 20.2 & 2.5 & 77.3 \\
\hline Hyperion & -84.7 & 22.7 & 118.2 & 9.4 & 18.9 & 98.1 \\
\hline Iapetus & -99.5 & 2.9 & 81.8 & 12.0 & 2.6 & 71.4 \\
\hline
\end{tabular}

